\title{
Role of imatinib in the management of early, operable, and advanced Gl stromal tumors (GISTs)
}

This article was published in the following Dove Press journal:

OncoTargets and Therapy

19 June 2009

Number of times this article has been viewed

\section{John T Vetto}

Division of Surgical Oncology, Oregon Health \& Science University and the OHSU-Knight Cancer Institute, Portland, Oregon, USA
Correspondence: John T Vetto, MD, FACS Professor of Surgery, Division of Surgical Oncology, Oregon Health \& Science University and the OHSU-Knight Cancer Institute, 3 I8I S.W. Sam Jackson Park Rd., Portland, Oregon 97239, USA

$\mathrm{Tel}+\mathrm{I}(503) 494-550$ I

Fax + I 503-494-7573

Email vettoj@ohsu.edu

\begin{abstract}
Gastrointestinal stromal tumors (GISTs), the most common sarcoma of the GI tract, have unique kinase mutations that serve as targets for medical therapy. This article reviews the data supporting the use of the tyrosine kinase inhibitor (TKI) imatinib in GIST patients, and how this treatment should be combined with surgical resection (when possible) to optimize patient outcomes. Although surgical resection remains the mainstay of treatment for these tumors, patients with resected GISTs have high relapse rates that can be reduced by 1 year of adjuvant imatinib. Data also support the use of imatinib for patients with recurrent or unresectable GIST. In these patients the drug should be continued until progression, intolerance, or the patients are rendered resectable. Patients with advanced GIST who are successfully resected after imatinib treatment should be placed back on imatinib postoperatively. Patients who develop generalized progression (progression at 2 or more sites) on imatinib should move to other treatments, such as newer TKIs or other targeted approaches currently under study. Genotyping of the tumor should be considered in all pediatric GISTs and high risk adult GISTs, especially if there is progression on imatinib. Quality of life and the cost/benefit of new therapies are important issues for further study in patients with GIST.
\end{abstract}

Keywords: imatinib, GIST, gastrointestinal stromal tumors

\section{Background: GI stromal tumors and tyrosine kinase inhibitors}

Ramon y Cajal, a Spanish neuroanatomist and neurophysiologist, is credited with describing interstitial cells in Auerbach's plexus which have since been found to have both neural and stromal features by electron microscopy (EM). Now known as the Interstitial Cells of Cajal (ICC), these cells are thought to give rise to gastrointestinal stromal tumors (GISTs), either directly, or, alternatively, GIST and ICC may arise from a common mesenchymal stem cell. Like the ICC, GISTs have a classic immunohistochemical (IHC) staining pattern (positive for CD34, S-100, DES, keratin, negative for desmin), and, also like the ICC, GIST cells strongly express two kinases, c-kit and platelet-derived growth factor receptor (PDGF-R). In the case of GIST, 95\% express kit and approximately $7 \%$ express PDGF-R, usually in mutated forms. ${ }^{1}$

A skilled and meticulous medical illustrator, Cajal was awarded the 1913 Nobel Prize (which he shared with Camillo Golgi) for his work.

Until the unique EM and IHC features of ICC and GIST were elucidated, GISTs were previously misclassified as leiomyosarcomas or other spindle cell cancers. In fact, GISTs are very different pathologically and clinically. ${ }^{2}$ Importantly, GISTs are typically resistant to standard sarcoma adjuvant chemotherapy, with response rates 
(RRs) of $\sim 5 \%$, and no impact on survival. Similarly, radiation therapy (RT) offers mostly morbidity due in part to the fact that the intrabdominal location of these tumors limits its use; RT may have some role for rectal GISTS.

Rather, as outlined in this review, the high kinase expression in these tumors has allowed for treatment of these tumors with tyrosine kinase inhibitors (TKIs), ${ }^{3}$ which, when selectively combined with resection, has led to both a significant improvement in outcomes for this tumor and an explosive growth in our understanding of targeted therapy of solid tumors.

\section{Resection of primary GISTs: pre-operative considerations}

GIST may occur anywhere along the GI tract or elsewhere in the abdomen or retroperitoneum. Data from our and other institutions indicate that approximately $50 \%$ of primary GISTs are located in the stomach, $25 \%$ in the small bowel, and the rest are distributed in the colon, rectum, and

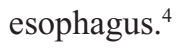

Some GISTs are found incidentally on imaging studies such as CT or endoscopic ultrasound (EUS) or in biopsies done for other reasons. Indeed, the management of these "microGISTs" is controversial. Tumors measuring $<1 \mathrm{~cm}$, commonly located in the stomach, have been found in $22.5 \%$ of autopsies of German adults older than $50,{ }^{5}$ and in $35 \%$ of Japanese gastrectomy specimens removed for other reasons. ${ }^{6}$ These small gastric GISTs are generally less aggressive than distal or larger tumors, and thus incidental gastric GISTs (usually found on endoscopy) can be followed with serial endoscopy and imaging as long as they remain asymptomatic. The management of incidental GISTs measuring 1 to $2 \mathrm{~cm}$ in size is more controversial. The mitotic rate of these tumors cannot be determined by fine needle aspiration, and therefore most surgeons would recommend resection, especially if it would involve minimal potential morbidity.

While all GISTs should be considered potentially malignant, nongastric GISTs are never considered "benign", and therefore most would favor resection of these lesions irrespective of size. ${ }^{7}$ Regardless, most GISTS are discovered on work-up for GI symptoms, and therefore are typically $>2 \mathrm{~cm}$ and/or symptomatic at the time of discovery. These lesions clearly require therapy.

The 2007 National Comprehensive Center Network (NCCN) GIST Guidelines state that the first step in the management of a potentially respectable GIST is to determine resectability with tests such as computed tomography (CT) and/or magnetic resonance imaging (MRI), chest imaging, endoscopic ultrasound (EUS), and endoscopy. We favor triple phase (porto-venous, early arterial, and late arterial) CT scanning because it best elucidates and rules out liver metastases, the most frequent site of metastases. Fluorodeoxyglucose-positron emission tomography (FDG-PET) is an excellent functional test that can be helpful in demonstrating tumor response to imatinib, but its role in determining resectability of primary GISTS is limited and it is usually not indicated. Importantly, the NCCN guidelines stress the role of a multidisciplinary GI program in assessing possible resection for primary GIST. If metastatic disease is ruled out by these tests preoperative biopsy of suspected GISTs is usually not indicated; the NCCN recommends biopsy only if the tumor is unresectable, if the diagnosis in doubt, or if neoadjuvant therapy is planned. ${ }^{8}$ If a biopsy is done EUS is usually the best method if the tumor is assessable endoscpically. ${ }^{3}$

Resectable GISTs should be resected - surgery remains the principal and only potentially curative therapy for localized, resectable primary GIST. ${ }^{7}$ After successful resection, the 2007 NCCN guidelines recommended postoperative imatinib if the surgeon removed all gross disease but the operative specimen reveals positive microscopic margins ( $\mathrm{R} 1$ resection) or if gross disease was left behind ( $\mathrm{R} 2$ resection): observation was recommended if an $\mathrm{R} 0$ resection (negative microscopic margins) was achieved. ${ }^{8}$ With the recent (December 2008) approval by the FDA for imatinib as adjuvant therapy for all GISTs, the issues have become somewhat more complex and will be discussed at the end of this review in light of the information that follows regarding the available data to date.

\section{Conduct and importance of surgical resection for primary GISTs}

Once in the operating room with a patient with an apparently resectable GIST, the surgeon should perform complete gross resection of the tumor with its pseudocapsule, using a "no-touch technique" as much as possible. This often requires segmental resection rather than peritumoral resection in order to achieve the best margins, within the limits of any retroperitoneal/intraperitoneal sarcoma resection (ie, margins are sometimes limited by adherence of the tumor to major vascular structures or nerves). Optimal resection is often assisted by the fact that like other sarcomas, GISTS are "pushers" rather than "invaders" and can often be lifted off surrounding organs; even lesions that appeared to be invading adjacent structures on preoperative scans are often found to be respectable at operation. That is, preoperative imaging can sometimes underestimate the resectability of tumors. 
Conversely, images may have missed small tumor implants that may render the tumor unresectable. The surgeon should look for such metastases by examining liver and peritoneal surfaces carefully and resecting them whenever possible. ${ }^{3,7}$

Adult GISTs do behave like other sarcomas in that they almost never spread to nodes; therefore routine lymph node dissection is not required. Similarly, extra-abdominal metastases and ascites are rare, facts that also assist with surgical resection. As alluded to above, it is essential to avoid tumor rupture and bleeding; GISTs are fragile tumors, and such events promote tumor dissemination and recurrence. Thus, the conduct of the resection of primary GISTs is critical to determining patient outcomes.

Several special issues regarding GIST resection deserve mention. First, it is unclear whether the surgeon should immediately return the patient to the operating room for a re-operation if the final microscopic margins are positive (R1 resection) when the surgeon expected an R0 report. This appears to vary by center, but is clearly also dependent on the findings at operation, patient factors, and surgeon and patient preference. There are no data that directly address this issue, although many centers now recommend starting imatinib instead of reoperation, with clinical follow-up. Secondly, esophageal GISTs may require either esophagectomy ${ }^{9}$ or enucleation (segmental resection is not an option in the esophagus). Which of these very different operative approaches is chosen depends on tumor, patient, and institutional factors. ${ }^{3}$ Thirdly, because half of all GISTS occur in the stomach, the surgeon frequently needs to decide on the extent of gastric resection. Most commonly, this can be segmental, with major gastrectomies reserved for GE junction or pylorus tumors. ${ }^{10}$

Finally, the use of laparoscopy deserves special mention. Although the 2004 NCCN guidelines listed GIST as a contraindication to laparoscopic resection, our and other institutions have been resecting GISTs safely using laparoscopic or laparoscopic-assisted techniques for some time. In 2006 Novitsky et al reported on 50 selected cases of primary GIST resected laparoscopically using careful techniques, including the use of specimen extraction bags and intra-operative ultrasound/endoscopy. Forty-seven patients had local resections and 3 had segmental resection. The mean operative time and average blood loss were very favorable (135 min and $85 \mathrm{~mL}$, respectively). At 36 months average follow-up, $92 \%$ of patients remained free of disease, and 4 had recurred, all with liver metastases (ie, recurrences were nature of disease and not local recurrences). ${ }^{11}$ This and other reports $^{3,12,13}$ emphasized that, as with any laparoscopic cancer resection, laparoscopic resection of GIST is safe as long as the surgeon adheres to oncologic principles, especially by avoiding direct instrumentation of tumor. Based on such reports, the $2007 \mathrm{NCCN}$ guidelines listed laparoscopic resection as an option for the treatment of primary GIST tumors. $^{8}$

The complete resection of a primary GIST is an important prognostic marker. The surgeon's goal should always be an $\mathrm{R} 0$ resection, as complete resection of GIST remains the best treatment even in the era of imatinib. ${ }^{3}$ Many tumors can be completely resected at presentation. After $\mathrm{R} 0$ resection, the most important prognostic factors are tumor factors, and include tumor size ( $<2 \mathrm{~cm}$, vs 2 to $5 \mathrm{~cm}$, vs 5 to 10 $\mathrm{cm}$, vs $>10 \mathrm{~cm}$ ), location (gastric best, small bowel worst), and tumor mitotic rate ( $<5$, vs 5 to 10 , vs $>10$ mitoses $[\mathrm{M}] / 50$ high powered fields [hpf]). ${ }^{14,15}$ Using these criteria, certain subgroups of GISTs (eg, larger tumors with higher numbers of mitoses) can be considered as "high risk", although-as will be stressed in the sections that follow-the definition of "high risk" can vary in the literature, among various institutions, and between different clinical trials. For example, at our institution the definition of "high risk" GISTs actually depends on the tumor site, which takes into account the importance of tumor location. ${ }^{4}$ Specifically, we define "high risk" for gastric GISTS as tumors $>10 \mathrm{~cm}$ and having $>5 \mathrm{M} / 50 \mathrm{hpf}$, and for GISTS distal to the stomach as those that are $>5 \mathrm{~cm}$ and having at least $5 \mathrm{M} / 50 \mathrm{hpf}$.

\section{Resected GIST: rationale for adjuvant imatinib}

While GISTs are usually treated up front with resection, R0 resection, as noted above, is clearly not the only predictor of patient outcome. Resected GISTs can have high recurrence rates, and prior to the availability of imatinib GIST patients had particularly high failure rates. Tumors $>10 \mathrm{~cm}$ in size were associated with 5-year disease-free survival (DFS) of only $20 \%$ and median times to progression (TTP) of 7 months to 2 years. Only $10 \%$ of patients remained disease-free after extended follow-up. ${ }^{16}$ Given this high recurrence rate and the existence of an effective oral drug with a low toxicity profile which targets a tyrosine kinase (TK) expressed in over $95 \%$ of tumors, it was only natural that clinical investigators turned research endeavors towards combining resection with systemic approaches using Imatinib.

Imatinib (Gleevec ${ }^{\circledR}$; Novartis) is a TKI which inhibits the Philadelphia chromosomal Bcr-Abl and the TK c-kit. It is also thought to induce apoptosis (see below). The most encouraging rationale for the use of this inhibitor in GIST 
were data from the Multicenter ("Pivotal") trial, ${ }^{17}$ Sarcoma Intergroup Trial, ${ }^{18}$ The European Organization for Research and Treatment of Cancer (EORTC)-Italian Sarcoma GroupAustralasian Gastrointestinal Trials Group, ${ }^{19}$ and other trials $^{3}$ of imatinib in patients with advanced (unresectable and metastatic) GIST. These studies demonstrated clinical benefit in over $80 \%$ of patients, with survival dramatically better than historical controls from the Southwest Oncology Group (SWOG) 8616 and 9627 trials. ${ }^{16}$

\section{Pharmacology of imatinib}

Imatinib, a 2-phenylaminopyrimidine derivative, is a specific inhibitor of a number of TK enzymes. It binds to the TK active site, leading to a decrease in kinase activity (Figure 1). Imatinib is specific for the TK domain in $a b l$ (the Abelson proto-oncogene), c-kit and PDGF-R. Imatinib is rapidly absorbed orally and is highly bioavailable: $98 \%$ of an oral dose reaches the bloodstream. Metabolism of imatinib occurs in the liver and is mediated by several isozymes of the cytochrome P450 system, including CYP3A4 and, to a lesser extent, CYP1A2, CYP2D6, CYP2C9, and CYP2C19. The main metabolite, $N$-demethylated piperazine derivative, is also active. The major routes of elimination are billiary and enteral; only a small portion of the drug is excreted in the urine. Most of imatinib is eliminated as metabolites: only $25 \%$ is eliminated unchanged. The half-lives of imatinib and its main metabolite are 18 and 40 hours, respectively. ${ }^{20}$

\section{Resected GIST: adjuvant trials/efficacy studies}

Based on the above mentioned encouraging data on imatinib in advanced GIST, the American College of Surgeons Oncology Group (ACoSOG) embarked on 2 adjuvant trials of imatinib after resection of primary GIST - a nonrandomized

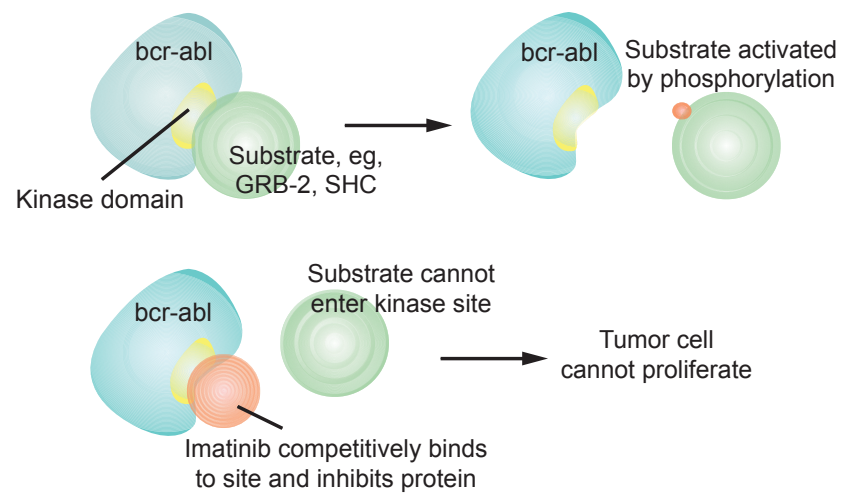

Figure I Mechanism of action of imatinib (source: http:Ilwikipedia/imatnib [accessed March 2, 2009]). phase II administration of imatinib to patients with high risk GIST (ACoSOG Z9000), and a placebo-controlled, randomized study of imatinib for more intermediate-risk patients (ACoSOG Z9001). ${ }^{\text {? }}$

The primary objective of the Z9000 trial was overall survival (OS) after administration of $400 \mathrm{mg}$ /day for 1 year of the drug to patients after resection of high risk GIST; the secondary objectives were 2- and 5-year recurrence and toxicity. To be included in the trial patients needed to be imatinib-naïve, have tumors that were c-KIT positive, received no prior adjuvant therapy, and undergo at least an R1 resection with no residual disease on post-operative imaging. "High risk" for this trial was defined as any of the following: tumors at least $10 \mathrm{~cm}$ in greatest dimension, the presence of tumor rupture before or during operation, intraperitoneal hemorrhage, or the finding at operation of multifocal intraperitoneal tumors (all resected); ie, number of mitoses was not included in the definition of high risk.

The 4-year results of this trial were presented by DeMatteo et al in 2008. Among the 107 eligible patients, imatinib was started at a median of 59 (range 25 to 84 ) days after operation. Patients had a median age of 58 years (range 19 to 79) and a median tumor size of $13 \mathrm{~cm}$ (range 3 to 42 ). Fifty percent of the tumors were gastric and $42 \%$ were small intestine. Imatinib therapy was well tolerated (previously reported in 2005$)^{21}$. At a the median follow-up of 4 years the $1-, 2-$, and 3 -year OS were $99 \%, 97 \%$, and $97 \%$, respectively, and the 1-, 2-, and 3-year relapse-free survivals (RFS) were $94 \%, 73 \%$, and $61 \%$, respectively. The authors concluded that imatinib $400 \mathrm{mg}$ for 1 year after resection of highrisk primary GIST prolonged RFS and OS compared with historical controls. ${ }^{22}$

The primary objective of the Z9001 trial was to determine the OS of patients with GIST treated with imatinib (again, $400 \mathrm{mg}$ /day for 1 year) in an adjuvant setting relative to placebo. The secondary objectives were RFS and safety/efficacy in an adjuvant setting. This trial included all patients with GISTS $\geq 3 \mathrm{~cm}$, resected within 70 days prior to registration, who had kit-positive tumors, were imatinib-naïve, and had no prior adjuvant therapy.

The preliminary results of this trial were presented by DeMatteo et al in 2007. At a median follow-up of 1.2 years, the 1-year RFS among the 708 completely resected patients was $97 \%$ for the imatinib arm, and $83 \%$ for placebo. This difference reached statistical significance for tumors $>6 \mathrm{~cm}$. No effect on OS has yet been seen; 10-year survival follow-up is ongoing. ${ }^{23}$

Based on these and other trials, imatinib was FDA approved in December 2008 for the adjuvant treatment of all 
resected primary GISTs. Ongoing trials continue to address the question of adjuvant imatinib, including a study from the EORTC (for GIST $>3 \mathrm{~cm}$, randomizing 2 years of imatinib vs placebo), and a trial from the Scandinavian Sarcoma Group (randomizing between 12 vs 36 months of treatment in high risk GIST patients). ${ }^{7}$

Overtreatment of GIST patients with adjuvant imatinib, an expensive drug with known toxicity, is an obvious concern for a number of reasons. First, as noted above, the randomized trials leading to FDA approval included only those GISTs $>3 \mathrm{~cm}$, with a statistically significant improvement in RFS only for tumors $>6 \mathrm{~cm}$. Secondly, the data as yet show no improvement in OS. Thirdly, the ACoSOG studies were not stratified by mitotic rate, now known to be a key prognostic factor. Finally, small, good prognosis GISTS may be cured with surgical resection alone-although at present there are no definite markers to identify these patients.

\section{Safety and tolerability of imatinib in GIST patients}

Common reactions reported with imatinib include fever, headaches, fluid retention (peripheral and periorbital edema), nausea and vomiting, dyspepsia, muscle cramps and pain, arthralgias, diarrhea, hemorrhage and anemia, neutropenia, upper respiratory infections, and elevated liver transaminases and bilirubin. Patients receiving imatinib should be monitored with liver function tests and consideration should be given for baseline troponins and electrocardiogram if they are being treated for hematologic disorders, and thyroid function tests if they have had a thyroidectomy.

Early results from the ACoSOG included a 2005 report by DeMatteo et al regarding the safety and tolerability of imatinib in patients with GIST; given orally $400 \mathrm{mg}$ /daily for 1 year, the drug was well tolerated. No grade 4 or 5 toxicity was seen. Nineteen (17\%) patients had grade 3 toxicity, consisting of neutropenia $(2 \%)$, dermatitis $(2 \%)$, and increased ALT (2\%). The most frequent toxicities of any grade included edema (55\%), fatigue (43\%), nausea (42\%), diarrhea (42\%), and dermatitis (27\%). Eighty-seven (82\%) patients completed the 1 year of imatinib, and $72(68 \%)$ tolerated full dose without a dose reduction. ${ }^{21}$

Rare but serious reactions reported with imatinib include liver failure (ascites, anasarca, hepatotoxicity), left ventricular dysfunction (pulmonary edema, pleural effusions, congestive heart failure [CHF], pericardial effusions), thrombocytopenia and bleeding (GI hemorrhage, anemia), neutropenia, exfoliative dermatitis, hypokalemia, hypothyroidism, and - very rarely - Stevens-Johnson syndrome and erythema multiforme.
As with any TKI, imatinib should be used with caution in patients with hypersensitivity to TKIs, cardiac risk factors, or impaired liver function, as the drug is extensively metabolized in the liver; only $12 \%$ is renally excreted. ${ }^{20}$ Patients should avoid pregnancy and breast feeding.

Rarely, patients with advanced GIST on TKI therapy may develop complications such as intraluminal or intraperitoneal hemorrhage, rupture, abscess, fistula, or obstruction, necessitating emergency operation. All 3 operative deaths in one series occurred in patients undergoing emergency surgery. ${ }^{24}$ Accordingly, pre-emptive operation should be considered in patients with evidence of fistulization, ongoing necrosis, or limited hemorrhage. ${ }^{7}$

\section{Advanced GIST: rationale for neoadjuvant and palliative imatinib}

As mentioned above, imatinib was first tested in patients with advanced GIST. The rationale for this testing was clear: 95\% of GIST express mutated c-KIT, and operative therapy alone for advanced GIST, as mentioned above, would be expected to fail in the majority of cases. Also as mentioned previously, subsequent trials, especially the Pivotal Trial, demonstrated that over $80 \%$ of patients with advanced GIST derive some clinical benefit. ${ }^{17-19}$

Given these findings, imatinib has been applied to patients with operable GIST in one neoadjuvant trial, ${ }^{25}$ and to inoperable GIST in the hope of rendering the disease operable..$^{3,7,26}$ This combined medical-surgical approach in advanced GIST is based on the facts that there are few complete responses with imatinib alone in advanced disease, that responding lesions when biopsied/resected usually contain viable cells, data from other tumor types that cytoreduction may improve surgical outcomes, the general idea that recurrent GIST behaves like metastatic disease and therefore may best be treated with a multimodal approach, and that imatinib given preoperatively has the potential to increase resectability or reduce the extent of surgery. ${ }^{7}$

Data from the Pivotal Trial demonstrated that the median time to overall response in patients with advanced GIST treated with imatinib was 13 weeks, and that $80 \%$ (of the $80 \%$ of patients who responded) did so within 6 months of therapy. ${ }^{17}$ Conversely, it is rare to see incremental tumor shrinkage after 9 months, and the median time to progression on imatinib is approximately 2 years. ${ }^{27}$ Accordingly, centers using imatinib for advanced GIST in the hope of rendering patients resectable generally follow patients (who are responding) out on at least 6 months of therapy before 
considering surgical exploration, but usually operate before 24 months. $^{7}$

\section{Imatinib and surgery for advanced GIST: outcomes}

The Radiation Therapy Oncology Group (RTOG) 0132 trial was a phase II study of neoadjuvant/adjuvant imatinib given for advanced primary and metastatic/recurrent operable GIST. Imatinib was given to 52 patients at $600 \mathrm{mg} / \mathrm{day} ; 30$ patients had primary GIST (group A) and 22 had recurrent/metastatic GIST (group B). All were felt to be operable; 7\% of group A and $4.5 \%$ of group B had partial preoperative responses to imatinib, while $4.5 \%$ of group B progressed. The remainder had stable disease. Two-year PFS and OS were $83 \%$ and $93 \%$, for Group A, and 77\% and 91\% for Group B, respectively. Complications of operation and toxicity of imatinib were judged to be minimal. Accordingly, the authors concluded that neoadjuvant imatinib is feasible, and not associated with notable postoperative complications. ${ }^{25}$

One of the issues with this trial is that patients were evaluated for radiographic response to imatinib using the Response Evaluation in Solid Tumors (RECIST) criteria. These criteria, which are based on size changes on anatomic imaging, can under call response of GIST tumors to imatinib because 1) response, especially early on, can consist mostly of "dynamic" changes in the tumor (eg, decreased FDG-PET uptake and decreased tumor density on contrast-enhanced CT [CE-CT] scans), and 2) some responding GIST tumors may actually swell, a situation which may be labeled as progression by RECIST criteria if the increase in size of the sum of the greatest diameters of the tumors exceeds $20 \%$. Accordingly, many oncologists favor the newer Choi Criteria for radiographic evaluation of GIST response to therapy, which include a dynamic measure of response (a 15\% decrease in tumor density on CE-CT). ${ }^{28}$

The data on combined medical-surgical therapy for advanced and inoperable (or "possibly operable") GIST at present consists of small series; these studies report response rates a high as $76 \%$, similar to the findings of the Pivotal Trial. Approximately $25 \%$ of unresectable primary and recurrent/metastatic lesions convert to resectable, and long term disease control has been documented.,27,30 The timing of resection can be tricky; surgeons generally want to wait until the patient has had a maximum response to TKI therapy but this cannot always be known in foresight-the minimum 6 months of TKI therapy is usually given as discussed in the previous section. After resection most surgeons resume TKI post op; in the French Sarcoma Group and RTOG S-0132 studies patients recurred quickly if the imatinib was stopped postoperatively. ${ }^{7,25,31}$

Six studies to date have provided surgical outcomes data for patients with advanced GIST going to operation after imatinib. Of patients who get resected, $48 \%$ to $91 \%$ have $\mathrm{R} 0$ or R1 resections, with documented improvement in survival. However, these resections have been done in specialty centers with highly selected patients using an aggressive approach (Figure 2); 60\% involved peritonectomy/omentectomy, 60\% required multivisceral resections, and $40 \%$ involved resection of liver metastases. . $^{2,27,29,32-34}$

Two-thirds of patients with metastatic GIST develop liver metastases at some time during their course, and the liver can often be the only site of metastatic disease. Such liver lesions are often multifocal and may require multiple or repeated medical and surgical interventions to render the liver without evidence of disease, including radiofrequency ablation and hepatic artery embolization. ${ }^{35}$

One of the largest series of surgical outcomes using this approach was reported by Raut and colleagues at the Brigham and Women's Hospital in Boston. These authors reported their surgical results in 69 consecutive patients in terms of the preoperative response (on imaging) to imatinib (Table 1), categorizing responses as "stable disease" (no progression of the index lesions by the time of operation), "limited progression" (progression of 1 target), or "generalized progression" (progression of more than 1 target lesion). $\mathrm{R} 0$ or $\mathrm{R} 1$ resections

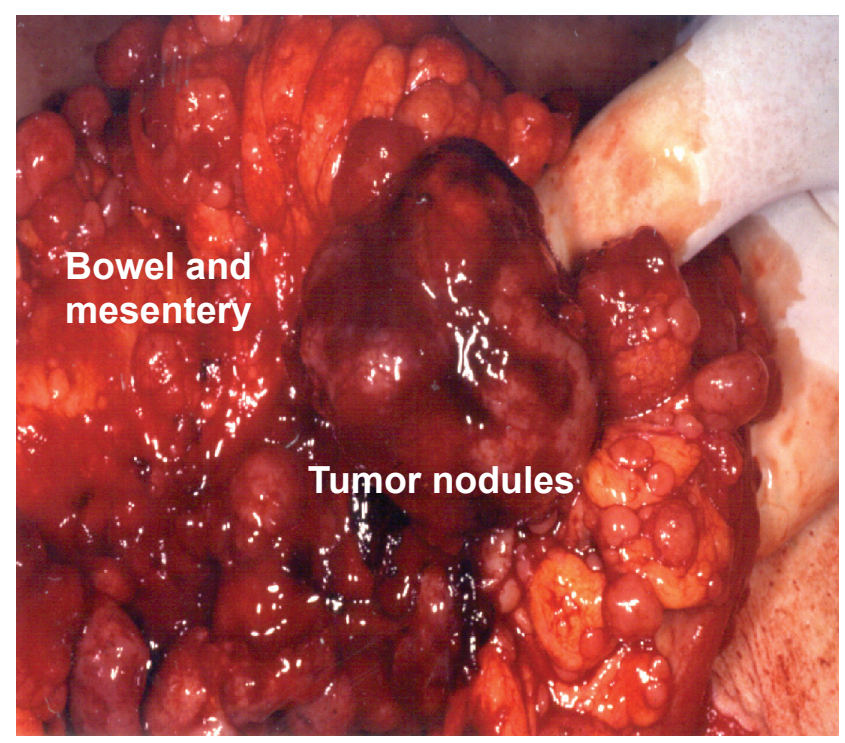

Figure 2 Intraoperative photograph of advanced GIST after imatinib therapy showing multiple tumor nodules on the bowel and mesentery despite a good radiographic response. Resection of this disease requires an aggressive approach, which frequently includes peritonectomy/omentectomy, multivisceral resections, and resection of liver (courtesy Dr C Corless). 
Table I Outcomes of resection in patients with advanced GIST on tyrosine kinase inhibitors $(n=69)$

\begin{tabular}{llll}
\hline & $\begin{array}{l}\text { Stable } \\
\text { disease }\end{array}$ & $\begin{array}{l}\text { Limited } \\
\text { progression }\end{array}$ & $\begin{array}{l}\text { Generalized } \\
\text { progression }\end{array}$ \\
\hline R0-RI & $78 \%$ & $25 \%$ & $7 \%$ \\
R2 & $4 \%$ & $16 \%$ & $43 \%$ \\
I-year PFS & $80 \%$ & $33 \%$ & $0 \%$ \\
I-year OS & $95 \%$ & $86 \%$ & $0 \%$ \\
\hline
\end{tabular}

Adapted with permission from Raut CP, Posner M, Desai J, et al. Surgical management of advanced gastrointestinal stromal tumorsafter treatment with targeted systemic therapy using kinase inhibitors. J Clin Oncol. 2006;24:2325-31. ${ }^{32}$ Copyright (C) 2006 American Society of Clinical Oncology.

Abbreviations: R0-RI, complete resection of all microscopic or macroscopic disease, respectively; R2, gross residual disease left behind; PFS, progression-free survival; OS, overall survival.

were accomplished in the majority of stable disease cases, whereas in almost half of generalized progression patients all gross disease could not resected. At 1 year the progression free and overall survival were high for stable disease patients, but all generalized disease had died of their tumors. The authors concluded that resection on imatinib is reasonable unless patients have generalized progression. ${ }^{32}$

\section{Imatinib-resistant GIST: options}

For patients progressing on imatinib, options include the multitarget TKI sunitinib (Sutent ${ }^{\circledR}$; Pfizer), usually given orally at $37.5 \mathrm{mg} /$ day. This drug primarily targets kit exon 9 mutants, forms of GIST that often do not respond to $400 \mathrm{mg}$ /day imatinib. ${ }^{36}$ Studies of newer TKIs, such as dasatinib and nilotinib (which can act through signaling pathways other than c-KIT and PDGF-R), ${ }^{37}$ are currently ongoing, as are studies with IPI-504, a heatshock protein 90 inhibitor. Work in GIST cell lines suggests that treatment with dasatinib or IPI-504 may provide a therapeutic alternative for GIST patients whose tumors carry the imatinib-resistant PDGR-F(D842V) mutant isoform. ${ }^{38}$

RAD001, an mTOR inhibitor, is currently in trial given in combination with imatinib in imatinib-resistant GISTs. The use of imatinib combined with chemo agents can be considered, based on evidence from CML patients. . $39-41^{-1}$

\section{GIST genotyping: current recommendations}

For all patients with GIST, especially those who develop advanced and/or imatinib resistant disease, the questions of whether and when to genotype the tumor (ie, perform mutational analysis) usually arise. The most recent (2007) NCCN guidelines recommend genotyping "ALL high risk and malignant (ie, advanced) GISTs". ${ }^{8}$
In fact, the vast majority of GIST tumors have a kit mutation, and most of these are at exon 11. Exon 11 mutations are usually associated with tumors that are located in the stomach, have a more favorable outcome, and initially respond to imatinib, although they may fail later. Fewer tumors have exon 9 mutations; these mutations are associated with nongastric sites, a less favorable prognosis, and respond less to imatinib at $400 \mathrm{mg} /$ day - they may respond to $800 \mathrm{mg}$ doses. Least favorable, and fortunately less common, are tumors with no kit mutation ("wild type"). These fail earlier and do not typically respond to TKIs. Pediatric GISTs are commonly wild type and this is reflected clinically by their aggressive morphology - these tumors are often multifocal, nodular, and node-positive. Specific gene mutations, such as codon 557-558 deletion and hTERT overexpression also mark high risk tumors. ${ }^{16,32}$

At our center, therefore, we do perform genotyping for specific indications where the information can be used clinically. We genotype all pediatric GISTs; while most are wild type and will do poorly on TKIs, a few have adult-type genotypes (exon 11 and 9 mutations) and TKIs may be indicated. The knowledge that a tumor expresses an exon 9 mutation marks cases where $800 \mathrm{mg} /$ day of imatinib should be tried. For patients who are either being considered for or are already failing sunitinib, some mutations, such as the novel kit exon 16 mutation L783V, predict clinical sunitinib resistance and mark patients who may not respond to this drug. Secondary mutations of the kit activation loop confer cross-resistance to both imatinib and sunitinib, and mark patients for whom TKIs may not be effective. Some imatinib resistant tumors express mutated PDGF-R and may be targets for PDGF-R inhibitors. ${ }^{4,41}$

\section{Patient-focused perspectives: quality of life, patient satisfaction, and support}

The advent of targeted therapy has converted high risk and metastatic GIST from a deadly disease to one where the chief issues are disease control, timing of therapies, and quality of life (QOL). Accordingly, support groups for patients facing therapy for GIST have appeared and have a very real role in advocacy for affected patients and their caregivers. Two of the largest include GIST Support International (www. gistsupport.org), a world wide organization of patients and caregivers which provides information and one-time grants for patient treatment, and Life Raft Group (www. liferaftgroup.org), a 501c national group with local chapters, which provides information, support, and advocacy. 
Because the health-related QOL and economic burden of GIST are timely from a payer, provider and patient perspective and may provide guidance for treatment decision making and reimbursement, it is not surprising that an extensive literature on these topics has appeared. Reddy and colleagues performed a systematic literature review of PubMed and 5 scientific meeting databases to identify and review 34 published studies and abstracts describing the epidemiologic, QOL and economic impact of GIST. Meta-analysis of these publications revealed that on the Functional Assessment of Chronic Therapy-fatigue (FACT-f) instrument, GIST patients scored 40.0 compared with 37.6 in anemic cancer patients $(0=$ worst; $52=$ least fatigue). Reported total costs over 10 years for managing GIST patients with molecular targeted therapy averaged approximately $£ 47,521$ to $£ 56,146$ per patient compared with $£ 4,047$ to $£ 4,230$ per patient with best supportive care. They concluded from their review that the health-related QOL burden of GIST is similar to that with other cancers, and the value of new therapies in GIST need to consider not only cost but also anticipated benefits and the unmet medical need in this condition. ${ }^{42}$

\section{Summary: the place of surgery and targeted therapy in the treatment of GIST}

GISTs, the most common sarcoma of the GI tract, have unique kinase mutations that serve as targets for medical therapy. Although an R0 surgical resection (microscopic negative specimen margins) remains the mainstay of treatment for these tumors, patients with resected GISTs have high relapse rates that can be reduced by 1 year of adjuvant imatinib. Data from studies supporting the use of imatinib in the adjuvant setting are still maturing, and the appropriate patient subsets and optimal duration of treatment are still unknown. For patients undergoing an $\mathrm{R} 1$ resection (tumor at 1 or more microscopic margins) options include either re-resection or imatinib, depending on the clinical setting.

Data clearly support the use of imatinib for patients with recurrent or unresectable GIST. In these patients the drug should be continued until progression, intolerance, or the patients are rendered resectable. Patients with advanced GIST who are successfully resected (R0 or R1) after imatinib treatment should be placed back on imatinib post-operatively. For patients who progress on $400 \mathrm{mg}$ imatinib, the dose can be increased to $800 \mathrm{mg} /$ day. Patients who develop generalized progression (progression at 2 or more sites) even on this higher dose should move to other treatments, such as newer
TKIs or other targeted approaches currently under study. Genotyping of the tumor should be considered in all pediatric GISTs and high risk adult GISTs, especially if there is progression on imatinib. QOL and cost/benefit of new therapies are important issues for further study in patients with GIST.

\section{Disclosures}

The author discloses no conflicts of interest.

\section{References}

1. Corless C, Fletcher JA, Heinrich MA, et al. Biology of gastrointestinal stromal tumors. J Clin Oncol. 2004;22:3814-3824.

2. Kirsch R, Gao ZH, Riddell R. Gastrointestinal stromal tumors: diagnostic challenges and practical approach to differential diagnosis. Adv Anat Pathol. 2007;14:261-285.

3. Hueman MT, Schulick RD. Management of gastrointestinal stromal tumors. Surg Clin NA. 2008;88:599-614.

4. Rubin BP, Heinrich MC, Corless CL. Gastrointestinal stromal tumour. Lancet. 2007;369:1731-1741.

5. Agaimy A, Wunsch PH, Hofstaedter F, et al. Minute gastric schlerosing stromal tumors (GIST tumorlets) are common in adults and frequently show c-KIT mutations. Am J Surg Pathol. 2007;31:113-120.

6. Kawanowa K, Sakuma Y, Sakurai S, et al. High incidence of microscopic gastrointestinal stromal tumors in the stomach. Human Pathol. 2006;37:1527-1535.

7. Raut CP, Ashley SW. How I do it: management of gastrointestinal stromal tumors. J Gastrointest Surg. 2008;12:1592-1599.

8. NCCN Clinical Practice Guidelines in Oncology: Soft Tissue Sarcoma, V.2.2007. Available at: http://www.globalgist.org/docs/NCCN guidelines.pdf (accessed March 5th, 2009).

9. Blum MG, Bilimoria KY, Wayne JD, et al. Surgical considerations for the management and resection of esophageal gastrointestinal tumors. Ann Thor Surg. 2007;84:1717-1723.

10. Wayne JD, Bell RH Jr. Limited gastric resection. Surg Clin NA. 2005;85:1009-1020.

11. Novitsky YM. Ketcher KW, Sing RF, et al. Long-term outcomes of laparoscopic resection of gastrointestinal stromal tumors. Ann Surg. 2006;243:738-745.

12. Choi SM, Kim MC, Jung GC GJ, et al. Laparoscopic wedge resection for gastric GIST: long-term follow-up results. Eur J Surg Oncol. 2007;33:444-447.

13. Nishimura J, Nakajima K, Omori T, et al. Surgical strategy for gastrointestinal stromal tumors: laparoscopic vs. open resection. Surg Endosc. 205;19:1109-1111.

14. DeMatteo RP, Gold JS, Saran L, et al. Tumor mitotic rate, size, and location independently predict recurrence after resection of primary gastrointestinal stromal tumor (GIST). Cancer. 2008:112:608.

15. Fletcher CDM. Berman JJ, Corless C, et al. Diagnosis of gastrointestinal stromal tumors: a consensus approach. Human Pathol. 2002:33: 459-465.

16. Blanke CD, Corless CL. State-of-the art therapy for gastrointestinal stromal tumors. Cancer Invest. 2005;23:274-280.

17. Demetri GD, vonMehren M, Blanke CD, et al. Efficacy and safety of imatinib mesylate in advanced gastrointestinal stromal tumors. $N$ Engl J Med. 2002;347;472-480.

18. Benjamin RS, Rankin C, Fletcher C, et al. Phase III dose-randomized study of imatinib mesylate (STI571) for GIST: Intergroup S0033 early results. Proceedings of the American Society for Clinical Oncology 2003;22 (abstract 3271).

19. Verweij J, Casali PG, Zalcberg J, et al. Progression-free survival in gastrointestinal stromal tumors with high-dose imatinib; randomised trial. Lancet. 2004;364:1127-1134. 
20. Druker BJ, Lydon NB. Lessons learned from the development of an Abl tyrosine kinase inhibitor for chronic myelogenous leukemia. J Clin Invest. 2000;105:3-7.

21. DeMatteo RP. Antonescu CR, Chadaram V, et al. Adjuvant imatinib mesylate in patients with primary high risk gastrointestinal stromal tumor (GIST) following complete resection: safety results from the US Intergroup Phase II trial ACOSOG Z9000. Proceedings of the American Society for Clinical Oncology 2005;23(16S):9009.

22. DeMatteo RP, Owzar K, Antonescu CR, et al. Efficacy of adjuvant imatinib mesylate following complete resection of localized, primary gastrointestinal stromal tumor (GIST)at high risk of recurrence: the US Intergroup phase II trial ACOSOG Z9000. [Abstract]. American Society of Clinical Oncology 2008 Gastrointestinal Cancers Symposium, January 25-27, 2008, Orlando, FL. A-8, 2008.

23. DeMatteo RP, Owzar K, Maki RG, et al. Adjuvant imatinib mesylate increases recurrence-free survival (RFS) in patients with completely resected localized primary gastrointestinal stromal tumor (GIST):North American Intergroup Phase III trial ACOSOG Z9001. Proceedings of the American Society for Clinical Oncology 2007, Abstract 10079.

24. Bonvalot S, Eldweny $\mathrm{H}$, Pechoux CL, et al. Impact of surgery on advanced gastrointestinal stromal tumors (GIST) in the imatinib mesylate era. Ann Surg Oncol. 2006;13:1596-1603.

25. Eisenberg BL, Harris J, Blanke CD, et al. Phase II trial of neoadjuvant/ adjuvant imatinib mesylate (IM) for advanced primary and metastatic recurrent operable gastrointestinal stromal tumor (GIST): early results of RTOG 0132/ACRIN 6665. J Surg Oncol. 2008, Oct 21 (Epub ahead of print).

26. Demetri GD, Benjamin RS, Blanke CD, et al. NCCN Task Force report: management of patients with gastrointestinal stromal tumor (GIST)-update of the NCCN clinical practice guidelines. J Natl Compr Canc Netw. 2007:5 Suppl:S1-S29.

27. DeMatteo RP, Maki RG, Singer S, et al. Results of tyrosine kinase inhibitor therapy followed by surgical resection in metastatic gastrointestinal stromal tumor. Ann Surg. 2007;245:347-352.

28. Benjamin RS, Choi H, Macapinlac HA, et al. We should desist in using RECIST, at least in GIST. J Clin Oncol. 2009;25:1760-1764.

29. Andtbacka RH, Ng CH, Scaife CL, et al. Surgical resection of gastrointestinal tumors after treatment with imatinib. Ann Surg Oncol. 2007; 14:14-24.

30. Gutierrez JC, Perez EA, Moffat FL, et al. Should soft tissue sarcomas be treated at high volume centers? An analysis of 4205 patients. Ann Surg. 2007;245:952-958.
31. Blay JY, LeCesne A, Ray-Cloquard I, et al. Prospective multicentric randomized phase III study of imatinib in patients with advanced gastrointestinal stromal tumors comparing interruption versus continuation of treatment beyond 1 year: the French Sarcoma group. J Clin Oncol. 2007;25:1107-1113.

32. Raut CP, Posner M, Desai J, et al. Surgical management of advanced gastrointestinal stromal tumors after treatment with targeted systemic therapy using kinase inhibitors. J Clin Oncol. 2006;24:2325-2331.

33. Gronchi A, Fiore M, Miselli F, et al. Surgery of residual disease using molecular-targeted therapy with imatinib mesylate in advanced/ metastatic GIST. Ann Surg. 2007;245:245-346.

34. Rutkowski P, Nowecki Z, Nyckowski P, et al. Surgical treatment of patients with initially inoperable and/or metastatic gastrointestinal stromal tumors (GIST) during therapy with imatinib mesylate. J Surg Oncol. 2006;93:304-311.

35. Maehara N Chijiiwa K, Eto T, et al. Surgical treatment for gastric GIST with special reference to liver metastases. Hepatogastroenterology. 2008;55:512-516

36. Judson IR. Prognosis, imatinib dose, and benefit of sunitinib in GIST: knowing the genotype. J Clin Oncol. 2008;26:5322-5325.

37. Braconi C, Bracci R, Cellerino R. Molecular targets in Gastrointestinal Stromal Tumors (GIST) therapy. Curr Cancer Drug Targets. 2008;8:359-366.

38. Dewaele B, Wasag B, Cools J, et al. Activity of dasatinib, a dual SRC/ ABL kinase inhibitor, and IPI-504, a heat shock protein 90 inhibitor, against gastrointestinal stromal tumor-associated PDGFRAD842V mutation. Clin Cancer Res. 2008;15;14:5749-5758.

39. Kitamura Y. Gastrointestinal stromal tumors: past, present, and future J Gastroenterol. 2008;43:488-508.

40. Reichert P. Novel approaches to imatinib-resistant GIST. Curr Oncol Rep. 2008;10:344-349.

41. Heinrich MC, Corless CL, Liegl B, et al. Mechanisms of sunitinib malate resistance in gastrointestinal stromal tumors. Proceedings of the American Society for Clinical Oncology 2007;25(18S):10006 (Abstract), 656(S).

42. Reddy P, Boci K, Charbonneau C. The epidemiologic, health-related quality of life, and economic burden of gastrointestinal stromal tumours. J Clin Pharm Ther. 2007;32:557-565.
OncoTargets and Therapy

\section{Publish your work in this journal}

OncoTargets and Therapy is an international, peer-reviewed, open access journal focusing on the pathological basis of all cancers, potential targets for therapy and treatment protocols employed to improve the management of cancer patients. The journal also focuses on the impact of management programs and new therapeutic agents and protocols on

\section{Dovepress}

patient perspectives such as quality of life, adherence and satisfaction. The manuscript management system is completely online and includes a very quick and fair peer-review system, which is all easy to use. Visit http://www.dovepress.com/testimonials.php to read real quotes from published authors. 